\title{
Testing galaxy formation models with the GHOSTS survey: The stellar halo of M81
}

A. Monachesi ${ }^{1}$, E. Bell ${ }^{1}$, D. Radburn-Smith ${ }^{2}$, M. Vlajić ${ }^{3}$, R. de Jong ${ }^{3}$, J. Bailin ${ }^{1}$, J. Dalcanton ${ }^{2}$, B. Holwerda ${ }^{4}$ and D. Streich ${ }^{3}$

${ }^{1}$ Department of Astronomy, University of Michigan, 830 Dennison Bldg., 500 Church St., Ann Arbor, MI 48109, USA, ${ }^{2}$ Department of Astronomy, University of Washington, Seattle, WA 98195, USA, ${ }^{3}$ Leibniz-Institut für Astrophysik Potsdam, D-14482 Potsdam, Germany,

${ }^{4}$ ESTEC, Keplerlaan 1, 2200 AG Noordwijk, The Netherlands. Email: antonela@umich.edu

Abstract. The GHOSTS survey is the largest study to date of the resolved stellar populations in the outskirts of disk galaxies (Radburn-Smith et al. 2011). The sample currently consists of 16 nearby disk galaxies, whose outer disks and halos are imaged with the Hubble Space Telescope (HST). I will present new results obtained from the study of 19 GHOSTS fields in M81's outermost part. The observed fields probe the stellar halo of M81 out to projected distances of $\sim 50 \mathrm{kpc}$, an unprecedented distance for halo studies outside the Local Group. The 50\% completeness levels of the color magnitude diagrams are typically at 2.5 mag below the tip of the red giant branch. When considering only fields located at galactocentric radius $R>15 \mathrm{kpc}$, we detect no color gradient in the stellar halo of M81. We compare these results with model predictions for the colors of stellar halos formed purely via accretion of satellite galaxies (Bullock \& Johnston 2005). When we analyze the cosmologically motivated models in the same way as the HST data, we find that they predict no color gradient for the stellar halos, in good agreement with the observations (see Fig. 1).

Keywords. galaxies: halos, galaxies: individual (M81), galaxies: spiral, galaxies: formation
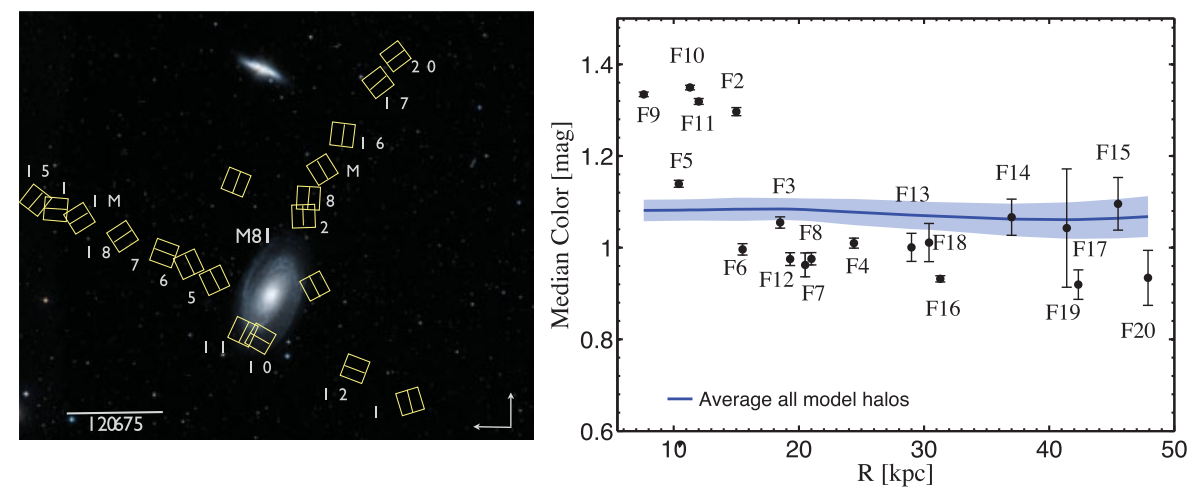

Figure 1. Left panel: Location of the 19 observed HST/ACS GHOSTS fields. Right panel: Median color profile of the M81 observed fields (dots). There is no color gradient for fields at $R>15.5 \mathrm{kpc}$, which we assume here to be M81's halo populations. The solid line shows the average color profile of the halo models analyzed and the shaded area indicates the $1 \sigma$ model-to-model deviations.

\section{References}

Bullock, J. S. \& Johnston, K. V., 2005, ApJ 635, 931

Radburn-Smith, D. J., et al. 2011, ApJS 195, 18 